\title{
Comparison of Registration Procedures of the Tibia in Robot-Assisted Total Knee Arthroplasty
}

\author{
Kathleen Denis ${ }^{1}$, Andrea Ranftl ${ }^{1}$, Geert Van Ham ${ }^{1}$, Jos Vander Sloten ${ }^{1}$, \\ Joris De Schutter ${ }^{2}$, Guy Fabry ${ }^{3}$, Johan Bellemans ${ }^{3}$ Remi Van Audekercke ${ }^{1}$, \\ and Georges Van der Perre ${ }^{1}$ \\ 1 Division of Biomechanics and Engineering Design, K.U.Leuven \\ Celestijnenlaan 200A, B-3001 Leuven, Belgium \\ 2 Division of Production engineering, Machine design and Automation \\ K.U.Leuven, Celestijnenlaan 300B, B-3001 Leuven, Belgium \\ 3 Department of Orthopaedics, U.Z.Leuven, Weligerveld 1 \\ B-3212 Pellenberg, Belgium
}

\begin{abstract}
The authors have implemented registration procedures by means of an intramedullary rod and by means of surface matching in their robot-assisted procedure for milling the tibia in TKA. Registration by means of an intramedullary rod is a simple and fast procedure that does not need CT-images. The accuracy is better than $2^{\circ}$ in the frontal plane. In case of a very deformed tibia or when the tibia is equipped with intramedullary nails from a previous operation however, it is not suitable. Registration using surface matching is an accurate and generally usable procedure. The difference between the predicted and the real orientation is less than $1.1^{\circ}$ in the frontal plane and less than $0.5^{\circ}$ in the sagittal plane. These registration procedures need immobilization of the tibia. Rigid immobilization is not necessary in case the registration is performed dynamically.
\end{abstract}

\section{Introduction}

\subsection{Robot-Assisted Procedure}

Robot-assisted surgery consists of three consecutive phases: the pre-operative planning phase, the registration phase and the phase of surgical action. The authors have developed a robot-assisted procedure for machining the tibia in total knee arthroplasty (TKA) 1 1 . In the planning phase, the surgeon chooses a tibial prosthesis and decides upon the ideal position and orientation of this prosthesis. Then, surgery starts with clamping the tibia in a bone holder. Before the planning of the operation can be transformed into robot motions, a registration procedure needs to be performed to determine the spatial relationship between the coordinate frames of the robot, of the anatomic object and of the pre-operative planning. After the tibia is located in the robot working space, the tibial condyles are removed conventionally. In the phase of surgical action the surgeon prepares the proximal tibia with the aid of the robot. At the same time 
the machining parameters can be monitored as additional information about the bone quality of the implantation bed [2]. Finally, the surgeon fixes the tibial component of the prosthesis on the obtained tibial plateau with the chosen fixation technique.

Figure 1 shows the experimental set-up with a 'Stäubli RX-130' robot provided with a six axis 'JR3' force sensor and a pneumatically driven mill. The tibia is clamped in a bone holder for the necessary fixation. Most commercial robotassisted systems for total knee arthroplasty mill the bone surfaces autonomously along a pre-operatively defined plan. In this way, the surgeon cannot deal with unexpected intra-operative events as fast as in the conventional operation. To overcome this disadvantage, and to control the robot with the forces and the moments of the surgeon, a hybrid force/velocity control strategy is used. The developed hybrid force/velocity control strategy is based on the task frame formalism for compliant motion tasks 34. In the current application, the velocity (respectively angular velocity) with which the mill moves in the planned cutting plane is proportional with the force (respectively moment) the surgeon exerts in that direction. The robot for his part constrains the motion of the mill to the predefined plane. In fact, the robot becomes a tool manipulated by the surgeon and constraining the degrees of freedom of the surgeon so that an accurate preparation of the bone surfaces is achieved. By using this force-control, the surgeon keeps the direct control of the operation: he decides where to mill with the robot, how fast the mill should proceed, etc. Milling experiments in laboratory circumstances showed that the accuracy is in the same order of magnitude as the accuracy of an autonomously milling robot.

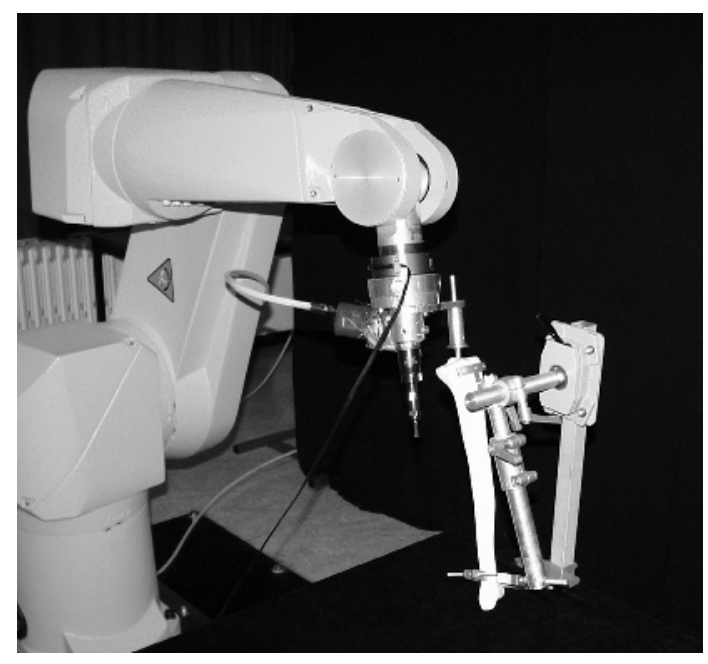

Fig. 1. Laboratory set-up with 'Stäubli RX-130' robot, 'JR3' force sensor and pneumatically driven mill. The tibia is clamped in the bone holder. A measurement tool is attached to the robot and is slid over a rod introduced in the medullary canal of the tibia. 


\subsection{Registration}

Since the registration determines the accuracy of the entire procedure, it is one of the key issues in robot-assisted surgery. Various procedures exist to perform the registration.

The first robot-assisted surgical procedures used artificial markers, so called fiducials 55. These artificial fiducials are to be placed pre-operatively on the anatomic object, in order to be visible on the pre-operative images. The planning is made in relation to these fiducials. In the registration phase, the robot touches the fiducials and measures in that way their position. This registration procedure is very accurate and fast, however, it has certain drawbacks: an extra operation with the risk of infections is needed to place the fiducials, and in addition the patient often suffers from post-operative pains. Therefore, other registration procedures have been developed.

Another registration procedure uses an application-dependent device. The principle of an application-dependent device is that the planning can be made in relation to the position and orientation of this device, and that its position and orientation can easily be detected during operation. An example is the intramedullary rod that can be placed in a unique way in the medullary canal of the tibia.

A third registration procedure uses surface matching. With this technique, points on the surface of the anatomic object are digitized during operation and are matched with the surface obtained from the pre-operative images.

The previous registration procedures are static, i.e. the tibia needs to stay immobilized during the further surgical action. Registering in real time the motion of the non-rigidly fixed tibia is called dynamic registration.

This paper discusses the two registration methods that have been developed and investigated, namely registration by means of an intramedullary rod and registration by means of surface matching, and it is also goes further into detail about dynamic registration.

\section{Registration by Means of an Intramedullary Rod}

\subsection{Procedure}

In case surgery is performed by means of an intramedullary rod, the surgeon plans the operation in the same way as in conventional surgery, on a frontal and a lateral roentgen image. He indicates on the images the desired orientation of the prosthesis with respect to the orientation of the mechanical axis. This mechanical axis connects the centre of the tibial plateau with the centre of the ankle. Its orientation corresponds to the intra-operative orientation of the intramedullary rod. During surgery, the surgeon introduces a rod in the medullary canal of the tibia. A handle and a measurement tool are attached to the robot in a unique way. Figure 1 shows the measurement tool in contact with the intramedullary rod in the tibia. The orientation of the intramedullary rod is measured using hybrid force/velocity control. Hence the surgeon leads the measurement tool to 
the rod and slides the measurement tool over the rod. He turns the tool until a marker on it points forward. In this way, the orientation of the rod is measured. The next step is the conversion of the planned angles in the lateral and in the frontal plane between the mechanical axis and the desired orientation of the bone cut to the robot. Then, the surgeon mills the tibial plateau with the aid of the robot.

\subsection{Accuracy}

The accuracy with which the intramedullary rod represents the mechanical axis is fundamental in the procedure. This accuracy was evaluated by measuring in 18 tibiae the difference in orientation between the rod and the mechanical axis. This was done using the RSA-technique (roentgenstereophotogrammetric analysis). Two insertion techniques, both commonly used in TKA, were applied: the centralized and the medialized insertion. For each tibia the following procedure was executed. A frontal and a sagittal radiograph were taken and the surgeon drew the mechanical axis on these radiographs. Then, the surgeon introduced an intramedullary rod in the tibia and a frontal and a sagittal radiograph of the tibia with the introduced rod were taken. By applying the RSA technique, the angle between the rod and the drawn mechanical axis was calculated. The experiments showed that the accuracy of this registration method does not depend significantly on the approach of insertion. The averaged difference in inclination between the rod and the mechanical axis is $0.21^{\circ}$ in the frontal plane $\left(\mathrm{s}=1.12^{\circ}\right)$ and $1.07^{\circ}$ in the sagittal plane $\left(\mathrm{s}=1.28^{\circ}\right)$ for the medialized approach [6].

\section{Registration by Means of Surface Matching}

\subsection{Procedure}

Another investigated registration method uses surface matching. The planning is made on pre-operative CT-images, which are necessary for the registration method. From those CT-images, the surface of the tibia is obtained. A set of points, called model points, is extracted from this surface. During operation, the robot digitizes points, the so-called data points, on the tibial surface. To this end, the robot is equipped with a registration tool (figure 2). After digitization, the data points are matched with the model points using the Iterative Closest Point (ICP) algorithm [7. The result of this algorithm is a translation $\mathrm{T}$ and a rotation $\mathrm{R}$ (figure [3), that give the mathematical relation between the model set and the data set. The planning also undergoes this transformation, and then the surgeon can mill the tibial surface with the aid of the robot.

\subsection{Accuracy}

To check the accuracy of this registration method, computer simulations and experiments on a composite tibia were performed. 
The computer simulations were performed using a real model set and a simulated data set. The model set was extracted from 1-mm slice thickness CT-images of a human tibia and consisted of 5802 proximal points and 878 distal points. The data set consisted of points randomly taken from the model set, with the addition of Gaussian noise (standard deviation $0.5 \mathrm{~mm}$ ) to their three coordinates to simulate the inaccuracies in reality. The mechanical axis and anteroposterior axis were indicated on the model set and were copied as evaluation axes to the data set. During the simulations, the evaluation axes moved together with the data set. After matching the data set on the model set using the ICP algorithm,

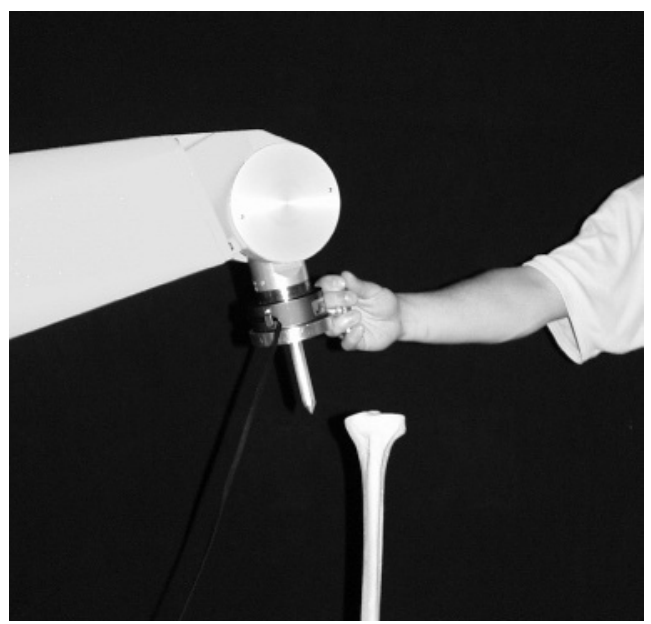

Fig. 2. The robot, equipped with the registration tool, approaches the tibial surface to digitize data points.

the orientation of the evaluation axes of the data set was compared with that of the model set. The simulations consisted of sets with different origin and number of data points. The data points originated either only from the proximal part of the tibia or also from the distal part. The number of data points ranged from 10 to 60 . It was important to keep the number of points as small as possible to limit the time consumption during operation since the data points are digitized intra-operatively. In each set, 25 simulations were done. The mean quadratic distance $\mathrm{D}^{2}$ between data points and the nearest model point was recorded in every simulation. Simulations without distal data points showed inaccuracies of more than $15^{\circ}$. Simulations with both proximal and distal data points showed an accuracy of better than $2^{\circ}$ in frontal and sagittal plane in 84 to $100 \%$ of the cases, depending on the number of data points. Sets with an omission of simulations with a high mean quadratic distance $\mathrm{D}^{2}$ showed an accuracy of better than $2^{\circ}$ in frontal and sagittal plane in all cases. The retained procedure has 25 proximal data points and 5 distal data points, and the registration is repeated if $\mathrm{D}^{2}$ is higher than $1.5 \mathrm{~mm}^{2}$. Table 1 summarizes the results for that set of simulations. 


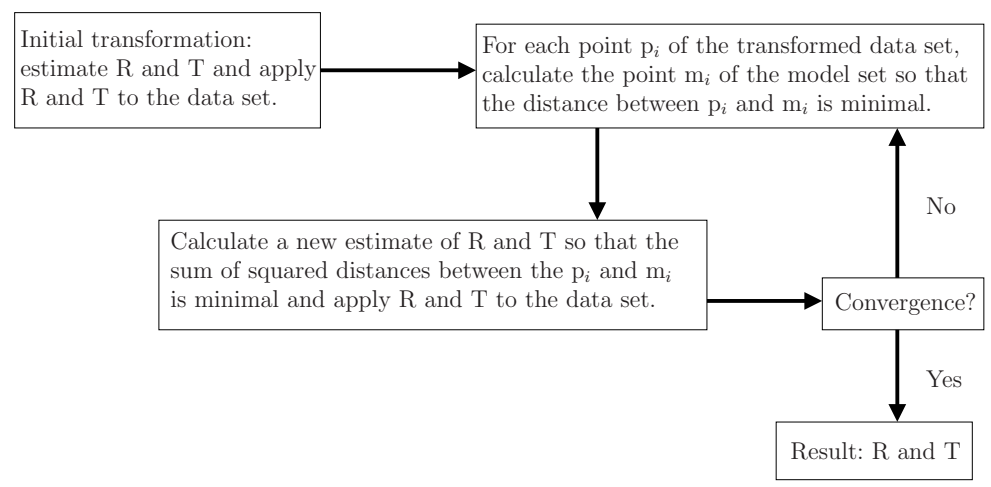

Fig. 3. Schematic overview of the iterative closest point algorithm; $\mathrm{p}_{i}$ is a point of the data set, $\mathrm{m}_{i}$ is a point of the model set, $\mathrm{i}$ is the number of data points.

Although these were computer simulations, the results can be seen as an upper limit for the results in reality. Firstly, because the noise added to the data points in the simulations was high enough and secondly, because the data points were taken randomly from the tibial surface. In reality, the data points can be chosen in an intelligent way. The registration procedure was used in laboratory experiments on a composite tibia (Sawbones), as shown in figure 2 The robot digitized 25 points on the proximal side and 5 on the distal side. The registration procedure was performed 25 times and $\mathrm{D}^{2}$ never exceeded $1.5 \mathrm{~mm}^{2}$. The standard deviation of the orientation of the mechanical axis and the anteroposterior axis of the tibia were stored (table 1). These results show that the results from the simulations are an upper limit for the results in practice.

Table 1. Mean and standard deviation of difference in orientation between evaluation axes of data and model set (for 25 simulations) and standard deviation for 25 experiments

\begin{tabular}{|l|l|l|l|}
\hline & $\begin{array}{l}\text { Mean difference } \\
\text { (from simulations) }\end{array}$ & $\begin{array}{l}\text { Standard deviation } \\
\text { (from simulations) }\end{array}$ & $\begin{array}{l}\text { Standard deviation } \\
\text { (from experiments) }\end{array}$ \\
\hline Frontal & $0.116^{\circ}$ & $0.45^{\circ}$ & $0.04^{\circ}$ \\
\hline Sagittal & $-0.007^{\circ}$ & $0.24^{\circ}$ & $0.03^{\circ}$ \\
\hline Transversal & $-0.363^{\circ}$ & $2.9^{\circ}$ & $0.60^{\circ}$ \\
\hline
\end{tabular}

\section{Dynamic Registration and Future Perspectives}

Robot-assisted TKA requires a full fixation of the tibia to assure high precision. This is done as mentioned above in section 1.1 via clamping the tibia. Thereby it is possible that tissues get harmed. If furthermore problems arise with the clamping during the operation the whole registration procedure has to be repeated. A possibility to avoid this intervention lies in the dynamic registration. Thereby sensor information of the position and movements of the tibia are fed 
back to the operating robot system. The robot system uses this information to compensate the spacial changes within the working area. The tibia has then still to be fixated but the invasive form of clamping can be avoided. A big challenge of this approach is to ensure that all the sensor data is provided to the robot system in real time. Though there are possibilities to use different sensor types the research will be focused in future to use vision data obtained by a camera system.

A quasi real-time set-up was developed with a robot-mounted camera system (SONY XC55bb CCD). The registration was done with the help of passive quadrangular markers. In the near future the experiments will be extended with high performance equipment.

In terms of robot control this kind of dynamic registration can be defined as tracking by visual servoing. In [8] it is stated that thereby the end-effector pose of the robot relative to a target or objects is controlled by vision. To realize and develop this control system former results on $2 \mathrm{D}$ contour following will be obtained from [9]. In this work already the interplay between different kinds of sensors (i.e. force and vision) has been realised.

This knowledge will be used in the near future to define and implement high level surgical tasks. These can be divided in sub-tasks which have to be coordinated and controlled (e.g. milling operation). With the help of the dynamic registration it will be possible to control and supervise the whole procedure either automatically or by the surgeon. The objective is that the robot will react "intelligently" on patient-individual environment and changes in the workspace.

\section{Conclusions}

Registration techniques that do not use pre-operative fiducials were developed. Two procedures for registration of the immobilized tibia in TKA have been investigated: registration by means of an intramedullary rod and registration by means of surface matching. Registration by means of an intramedullary rod is a simple and fast technique; there is no increase in operation time since this rod is also used in the conventional intervention. Moreover, it does not need the CTimages and it is an accurate technique, the difference between the predicted and the real orientation of the rod is less than $2^{\circ}$ in the frontal plane. This accuracy is sufficient for the presented application. However, this technique cannot be used in case of a severely bowed tibia, or in case the tibia is already equipped with prosthetic nails from a previous operation. Moreover, there is a small risk for fat embolism caused by the introduction of the rod in the bone. Therefore another registration technique, using surface matching, has been examined. This procedure uses CT-images. Distal data points are necessary in the data set. They can be obtained through a small incision at the ankle or they can be estimated on the skin. This is an accurate procedure; computer simulations show that for the established protocol the difference between the predicted and the real orientation is less than $1.1^{\circ}$ in the frontal plane and less than $0.5^{\circ}$ in the sagittal plane. Limiting the number of data points and choosing fast algorithms to perform the matching limits the intra-operative duration of the registration procedure. 
The registration procedures using pre-operative fiducials, using an intramedullary rod or surface matching all share one disadvantage: they are static registration procedures; this means they need the immobilization of the tibia. Thus if the immobilization is hampered during operation, the surgical action needs to be interrupted and the registration procedure needs to be repeated. Moreover, the immobilization can be invasive to the anatomic object. Dynamic registration could offer a solution: a camera system registers the position and orientation of the anatomic object in real time and this information is fed back to the robot system. A dynamic registration procedure is under development and will be implemented in the near future.

Acknowledgements. This research is supported by a grant of the Fund for Scientific Research-Flanders and by the grant number GOA/99/04. Geert Van Ham is supported as research assistant of the Fund for Scientific Research-Flanders.

\section{References}

1. G. Van Ham, K. Denis, J. Vander Sloten, R. Van Audekercke, G. Van der Perre, J. De Schutter, E. Aertbeliën, J. Bellemans, and G. Fabry. A force controlled robot as a surgical tool in total knee athroplasty. In 12th Computer Assisted Radiology and Surgery Congress, pages 699-704. Elsevier, 1998.

2. G. Van Ham, K. Denis, J. Vander Sloten, R. Van Audekercke, G. Van der Perre, J. De Schutter, E. Aertbeliën, S. De Mey, and J. Bellemans. Machining and accuracy studies for a tibial knee implant, using a force controlled robot. Computer Aided Surgery, 3: 123-133, 1998.

3. J. De Schutter and H. Van Brussel. Compliant robot motion I. A formalism for specifying compliant motion tasks. Robotics Research, 7(4): 3-17, 1988.

4. J. De Schutter and H. Van Brussel H. Compliant robot motion II. A control approach based on external control loops. Robotics Research, 7(4): 18-33, 1988.

5. T. C. Kienzle, S. D. Stulberg, M. Peshkin, A. Quaid, J. Lea, A. Goswami, and C. Wu. Total knee replacement: computer assisted surgical system uses a calibrated robot. IEEE Eng. Med. Biol., 14(3): 301-306, 1995.

6. K. Denis, G. Van Ham, J. Bellemans, L. Labey, J. Vander Sloten, R. Van Audekercke, G. Van der Perre, and J. De Schutter. How correctly does an intramedullary rod represent the longitudinal tibial axes? Clin. Orth. Rel. Res., 397: 424-433, 2002.

7. P. Besl and N. McKay. A method for registration of 3-d shapes. IEEE Transactions on pattern analysis and machine intelligence, 14(2): 239-256, 1992.

8. S. A. Hutchinson, G. D. Hager, and P. I. Corke. A Tutorial on Visual Servo Control. IEEE Trans. Robotics and Automation, 12(5): 651-670, October 1996.

9. J. Baeten. Integration of vision and force for robotic servoing. $\mathrm{PhD}$ thesis, Katholieke Universiteit Leuven, B-3001 Leuven, Belgium, December 2001. 